\title{
Hepatogenic Differentiation Capacity of Human Wharton's Jelly Mesenchymal Stem Cell in a Co-culturing System with Endothelial Cells in Matrigel/collagen Scaffold in the Presence of Fetal Liver Extract
}

\author{
Zahra Khodabandeh ${ }^{1,2}$, Zahra Vojdani ${ }^{1}$, Tahereh Talaei-Khozani ${ }^{1,3}$, Soghra Bahmanpour ${ }^{1}$ \\ ${ }^{1}$ Laboratory for Stem Cell Research, Department of Anatomy, School of Medicine, Shiraz University of Medical Sciences, Shiraz, \\ Iran, ${ }^{2}$ Stem Cell Technology Research Center, Shiraz Institute for Stem Cell and Regenerative Medicine, Shiraz University of Medical \\ Sciences, Shiraz, Iran, ${ }^{3}$ Tissue Engineering Lab, Department of Anatomy, School of Medicine, Shiraz University of Medical Sciences, \\ Shiraz, Iran
}

Background: Human Wharton's jelly mesenchymal stem cells (HWJMSCs) isolated from medical waste product can be considered as an accessible source of cells in regenerative medicine. Stem cell-derived hepatocytes have poor function and need appropriate niche to reconstruct the liver structure. Therefore, we attempted to find a novel approach in differentiating HWJMSCs into functional hepatic cells using 3D culture conditions and liver extract that recapitulates vital stage in liver development.

Materials and Methods: HWJMSCs were extracted from human Wharton's jelly, characterized by flow cytometry, and differentiated towards osteogenic and adipogenic lineages. HWJMSCs were co-cultured with HUVECs in 3D matrigel/collagen scaffolds in the presence of fetal liver extract for 14 days. The expression of specific liver genes were evaluated by lectins, PAS and immunocytochemistry.

Results: According to flow cytometry data, isolated cells from HWJMSCs were shown to express MSC markers. HWJMSCs co-cultured with HUVECs in matrigel/collagen scaffold with extract expressed albumin, lectins UEA and PNA. Immunohistochemistry of the cells in matrigel/collagen scaffold with or without extract exhibited a positive reaction for CK19.

Conclusions: Co-culturing of the HWJMSC/HUVEC in 3D matrigel/collagen scaffold is bimimicary of in vivo cell condition. The results showed that administration of the liver extract in $3 \mathrm{D}$ matrigel/collagen culture of HWJMSC/HUVEC can induce hepatocyte marker expression.

Keywords: Collagen, Matrigel, 3D culture, Hepatocyte, Liver extract

Accepted for publication June 17, 2017, Published online November 30, 2017

Correspondence to Zahra Vojdani

Department of Anatomy, Shiraz Medical School, Zand Street, 7134845794, Shiraz, Iran

Tel: +98-71-32304372, Fax: +98-71-32304372

E-mail: vojdaniz@sums.ac.ir

(a) This is an open-access article distributed under the terms of the Creative Commons Attribution Non-Commercial License (http://creativecommons.org/ licenses/by-nc/4.0/), which permits unrestricted non-commercial use, distribution, and reproduction in any medium, provided the original work is properly cited.

Copyright (c) 2017 by the Korean Society for Stem Cells Research

\section{Introduction}

During embryogenesis and regeneration, micro environmental cues regulate stem cell differentiation behavior and cell fate. Both neighboring cells and extracellular matrix $(\mathrm{ECM})$ constitutes a three dimensional framework as a natural niche for the stem, differentiating and fully differentiated cells $(1,2)$.

Mature and stem cell-derived hepatocyte functions decline by in vitro culturing due to the lack of natural cues 
and reconstruction of the physical and chemical properties of ECM has been reported to be important for maintaining the phenotype and function. Hence, culturing in 3D condition provides some of the natural micro environmental cues for hepatocytes (2). The long-term expansion of hepatocytes in vitro requires a different culture conditions including ECM environment together with a combination of growth factors for the expansion and differentiation of hepatocyte in 3D organoid model (3). 3D organoid structures can be especially used for obtaining human tissue in the future (4). One the most remarkable issue about liver organoids is the relation of endothelial cells with mesenchymal cells that can lead to angiogenesis (5).

Fetal liver ECM and growth factors play an important role in hepatocyte development and regeneration (6). It contains collagen, glycosaminoglycan (GAG) and various types of growth factors such as hepatocyte growth factor (HGF), fibroblast growth factor (FGF), interleukin 6 (IL-6), insulin growth factor (IGF) and vascular endothelial growth factor (VEGF) (7). These factors have been detected to increase hepatocyte organelles and albumin synthesis and also have an important role in hepatocyte differentiation and reconstruction of damaged liver (8).

In the last decade, natural hydrogels such as collagen, alginate and matrigel were used to improve differentiation efficiency and mature hepatocyte phenotype and function (9). Collagen is an essential component of ECM that plays a crucial role in differentiation, proliferation, migration and cell matrix interactions. Matrigel is a natural component which secrets from cultured Engelbreth-Holm-Swarm mouse tumor cell line and primarily consists of natural biopolymers laminin, collagen IV and entactin, as well as various growth factors $(10,11)$. These components can be used as scaffold, which recapitulate biophysical and biochemical environments for cells, and facilitates transferring of soluble signaling molecules, nutrients and metabolic wastes. Matrigel also provides mechanical integrity of fabricated tissue by absorbing compressive and tensile stresses (2). These scaffolds can also facilitate cell-matrix, -cell, and -growth factor interactions. Such a 3D culture system has been reported to enhance osteogenesis (12), and hematopoiesis (13). It also plays a role in hepatocyte proliferation, cell function improvement and establishing cell polarity compared with the conventional 2D culture. The hepatocyte polarity influences cell shape, cytoskeleton arrangement and distribution of organelles within cells, and the division of plasma membrane into three functionally different fields: basolateral, canalicular and lateral. The transport of small molecules and the metabolite ex- change with blood perform across the basal surface, whereas the secretion of bile acids and detoxification products take place on the apical surface (14).

Human Wharton's jelly mesenchymal stem cells (HWJMSCs) is abundant and accessible source of cells that can be considered as a good candidate to be used in regenerative medicine and bioengineering applications $(15,16)$.

Liver Sinusoidal endothelial cells (LSECs) play a critical effect on proliferation of hepatocytes. Le Couter et al., observed that the mice treated with VEGF-A showed an increase in proliferation of liver parenchymal cell and liver mass. It was also revealed that co-culture of primary hepatocyte with sinusoidal cell resulted to increase in hepatocyte proliferation. Activation of the VEGF receptor cause the sinusoidal endothelial cells to secrete a number of mitogenic factors, including HGF and IL6 $(17,18)$.

Based on these considerations, this study has attempted to demonstrate a new method for liver organoid engineering including reconstruction of the bile ducts and blood vessels using co-culture of HWJMSCs and Human umbilical vein endothelial cells (HUVECs) in the matrigel/collagen scaffolds.

\section{Materials and Methods}

\section{Primary culture of HWJMSCs}

Umbilical cords samples were collected from cesarean delivery of full-term infants after obtaining a written informed consent from parents. The tissue samples were transferred to the lab in cold Phosphate-Buffered Saline (PBS) containing $100 \mathrm{U} / \mathrm{mL}$ penicillin, $100 \mu \mathrm{g} / \mathrm{mL}$ streptomycin (Sigma Aldrich, UK) and washed three times. Then, both arteries were removed, the umbilical vein was opened, and the endothelium was crushed using a sterile blade. Then, the umbilical cords were cut into small explants about $5 \mathrm{~mm}$ each and they were placed in the dishes. After $15 \mathrm{~min}, \alpha$-MEM (Gibco BRL, life technology, Germany) containing 10\% Fetal Bovine Serum (FBS) (Gibco BRL), 1\% L-glutamine (Sigma Aldrich, UK), and $100 \mathrm{U} / \mathrm{mL}$ penicillin, $100 \mu \mathrm{g} / \mathrm{mL}$ streptomycin were added to the culture plates.

All procedures were approved by Shiraz University of Medical Sciences ethical committee.

\section{Cell characterization}

The cell suspension was adjusted at a concentration of $1 \times 10^{6}$ cells $/ \mathrm{mL}$ in $10 \% \mathrm{FBS} / \mathrm{PBS}$ as the blocking solution for $20 \mathrm{~min}$. Then, the cells were labeled with FITC-conjugated anti-CD44, CD90 and CD144, phycoerythrin-conjugated anti-CD34, CD 73 and CD106, and PerCP-con- 
jugated anti- CD105 antibodies (all from Abcam, UK) for $30 \mathrm{~min}$. The frequencies of positive cells were evaluated by a FACS calibrated instrument (BD, USA) and analyzed using FlowJo software (BD Biosciences).

\section{Fetal liver extract}

The human fetal liver extracts were obtained from human aborted fetus of 12 20 weeks after obtaining informed consent from their parents. The fetuses were transferred to the laboratory immediately after abortion, dissected and the liver removed. All steps were performed on ice.

The liver was cut into a dimension of $5 \mathrm{~mm}$, then the pieces of the livers were washed with PBS several times until the blood was removed completely. The pieces of liver were trypsinized and incubated at $37^{\circ} \mathrm{C}$ for $10 \mathrm{~min}$. The media containing $10 \%$ FBS were added to inactivate tryp$\mathrm{sin}$, and followed by centrifuging with $600 \mathrm{~g}$ for $10 \mathrm{~min}$ at $25^{\circ} \mathrm{C}$. The supernatants were removed, the residual of the tissues were snap-frozen in liquid nitrogen and the freeze and thaw was repeated 2 times and then the tissues were kept at -80 . After thawing on ice, the tissues were suspended in equal volume of lysis buffer (containing 1:10 a cocktail of protease inhibitor) for $45 \mathrm{~min}$ and homogenize using sonicator. Then the homogenized tissues were centrifuged with $15,000 \mathrm{~g}$ for $15 \mathrm{~min}$ at $4^{\circ} \mathrm{C}$. The supernatant were considered as the extracts were aliquoted at $15 \mathrm{~mL}$ volume and stored at -80 . Each aliquoted were lyophilized by freeze-dryer for $48 \mathrm{~h}$. The powders were kept at room temperature.

The concentration of proteins in an extract was measured using the dye-binding assay of Bradford.

\section{Bradford assay for protein quantification}

The procedure was based on the dye, Brilliant Blue G (Sigma-Aldrich) forms a complex solution with protein content of the fetal liver extract. The optical density of dye-protein complex was measured at $595 \mathrm{~nm}$.

The protein concentration was estimated by comparing the optical density with a series of known BSA solutions. The protein yield was $11 \mathrm{mg} / \mathrm{mL}$.

\section{Cytotoxicity assay}

HWJMSCs at a density of $5 \times 10^{4}$ cell/well for each 24 well plate were seeded, and then incubated with serial dilution of protein extract $(1 / 40,1 / 80,1 / 160,1 / 320,1 / 640)$ at $37^{\circ} \mathrm{C}$ for $48 \mathrm{~h}$. Thereafter, the media were removed and $1 \mathrm{~mL}$ of $0.005 \%$ neutral red was added and incubated at $37^{\circ} \mathrm{C}$ for $2 \mathrm{~h}$. The specimens were then fixed by formal calcium for $1 \mathrm{~min}$, and washed with PBS and incubated in acid alcohol for $2 \mathrm{~h}$ in a darkroom. The optical densities of the eluted solutions were measured at $540 \mathrm{~nm}$. Concentration that contained the most viable cells $(1 / 80)$ were chosen to be used in the next step.

\section{Matrigel/Collagen scaffold preparation \\ Collagen preparation: The working solution (1} $\mathrm{mg} / \mathrm{mL}$ ) was prepared from stock solution of collagen type I, rat-tail (3 mg/mL) (code A10483-01, Gibco). Ten times (10×) DMEM (Dulbecco's modified Eagle's medium) were mixed with collagen at 1:8 ratios on ice by slow and gentle pipetting. Then, $125 \mu \mathrm{L}$ of the working solution was added per well of 4-well plate and polymerized at $37^{\circ} \mathrm{C}$ for one hour. Thereafter, $1 \times 10^{3}$ cells were seeded per well.

Growth factor reduced matrigel (GFR-matrigel) (BD Biosciences, USA) was diluted in working collagen solution $(1 \mathrm{mg} / \mathrm{mL})$ at a ratio of $1: 3$.

\section{Differentiation of HWJMSCs in the Matrigel/Collagen scaffold}

Culture of HUVECs: Human umbilical vein endothelial cells (HUVECs) were cultured in the complete DMEM/F12 medium supplemented with $10 \%$ FBS, $100 \mathrm{U} / \mathrm{mL}$ penicillin, and $100 \mu \mathrm{g} / \mathrm{mL}$ streptomycin and incubated at $37^{\circ} \mathrm{C}$ in a $5 \% \mathrm{CO}_{2}$ to reach confluence.

Differentiation of HWJMSCs Co-culture with HUVECs in the Matrigel/Collagen scaffold: HWJMSCs in fourth passage were exposed to fetal liver extract in a conventional 2D culture condition for 7 days. On the day 7, HUVEC and HWJMSC were mixed at a ratio of $1: 3$ with matrigel/collagen solution and then incubated at $37^{\circ} \mathrm{C}$ to allow gel formation. After that the culture medium containing the fetal liver extract $(1 / 80)$ was added to the cells. The differentiation protocol was continued for 14 days and half of the culture medium was changed every 3 days.

Histological studies: The cell containing matrigel/collagen scaffolds were fixed in Bouin's solution and prepared histologically. Some sections were stained with H\&E and the rest were stained with Ulexeuropaeus (UEA) to detect endothelial cells and peanut agglutinin (PNA) to detect cholangiocytes. To do this, the slides were washed in Dulbecco's phosphate-buffered saline (DPBS), incubated with FITC-conjugated UEA and PNA (both at a dilution of $10 \mu \mathrm{g} / \mathrm{mL}$ ) for $2 \mathrm{~h}$ and then counterstained with 4', 6-diamidino-2-phenylindole (DAPI) immunostaining also was performed for cytokeratin 19 (CK19). Besides, some sections were incubated in blocking solution containing $5 \%$ goat serum in PBS and then in FITC-conjugated antiCK19 for $1 \mathrm{~h}$. Then, the sections were counterstained with DAPI. Finally, they were observed by fluorescence 
microscope (Olympus, BX51, and Japan). Some sections were also stained with PAS. To do this, the sections were incubated in $1 \%$ periodic acid for $5 \mathrm{~min}$ and then in Schiff for $15 \mathrm{~min}$.

\section{Statistical analysis}

All the statistical analyses were performed using GraphPad Prism 5 software (GraphPad, USA) and
Mann-Whitney test. p-value $<0.05$ was considered as statistically significant. All omitted experiments were performed in triplicate.

\section{Results}

\section{Characterization and differentiation of MSCs}

The flow cytometry analysis revealed that HWJMSCs

Table 1. The percentages of the WJ-MSCs showing Positive-reaction for CD markers by flow cytometry

\begin{tabular}{cccccccc}
\hline CD Markers & CD44 & CD90 & CD105 & CD106 & CD73 & CD34 & CD144 \\
\hline Percent & 89.4 & 90.9 & 55.2 & 30.5 & 96.2 & 2.58 & 3.13 \\
\hline
\end{tabular}

A
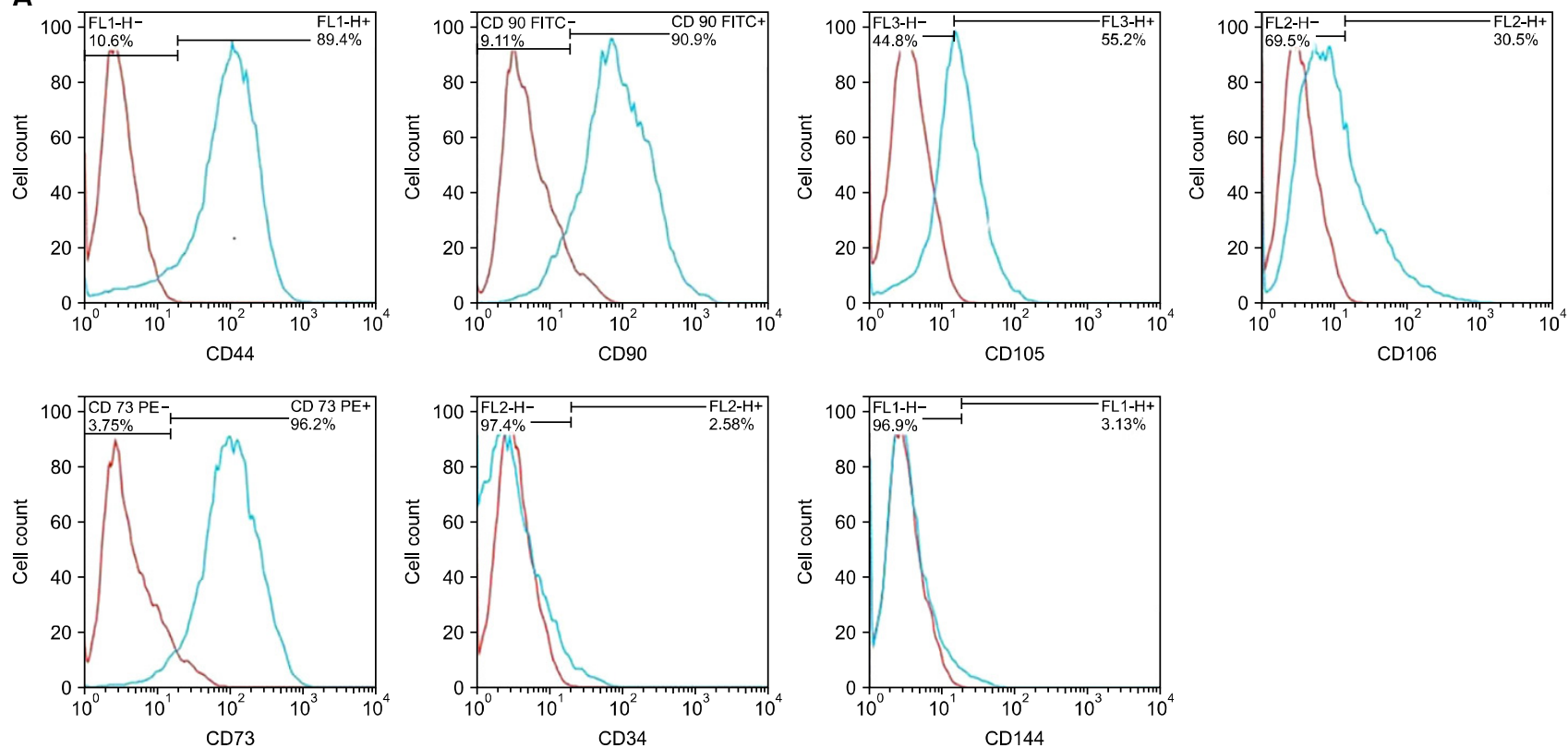

B

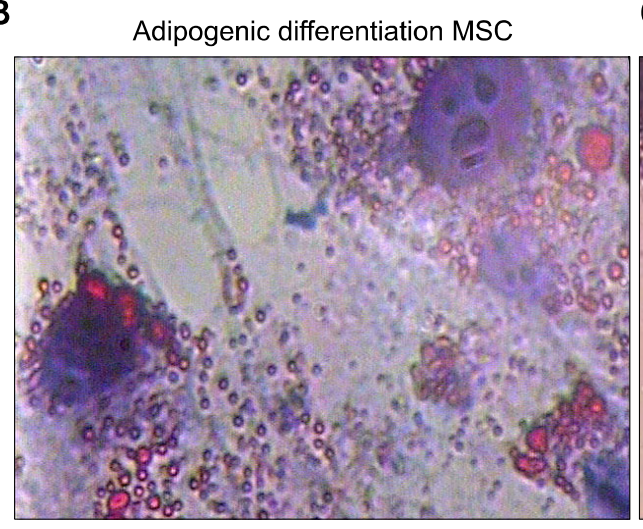

C Osteogenic differentiation MSC

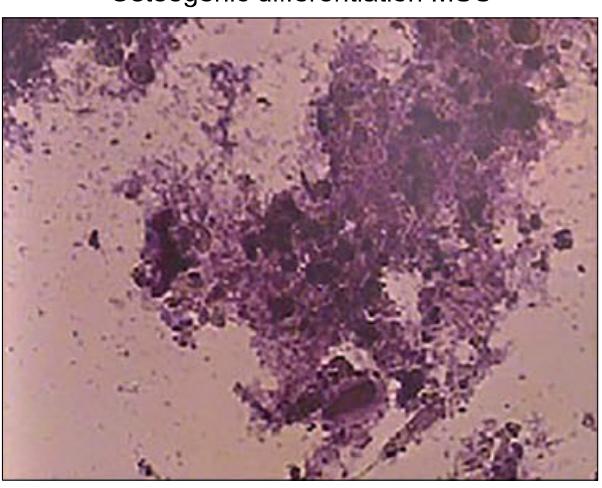

Fig. 1. The flow cytometry showed the frequency of omitted HWJMSCs which reacted to CD44, CD90, CD105, CD106 and CD73 was high; however, the frequency of the cells which reacted to CD34 and CD 144 was negligible (A). The blue line is positive cells, Oil red $\mathrm{O}$ staining showed that the cells stored lipid droplets in the presence of adipogenic medium (B), alizarin red $\mathrm{S}$ showed that the cells deposited $\mathrm{Ca}^{+}$in the presence of osteogenic medium (C). 
were positive for the MSC surface markers, such as CD44 (89.4\%), CD90 (90.9\%), CD105 (55.2\%), CD106 (30.5\%), CD73 (96.2\%), and negative for CD34 (2.58\%) and CD144 (3.13\%) markers (Table 1, Fig. 1).

In addition, the results of the alizarin red $S$ and oil red
O staining confirmed the capability of the MSC to differentiate into osteogenic and adipogenic cell lineages, respectively (Fig. 1).
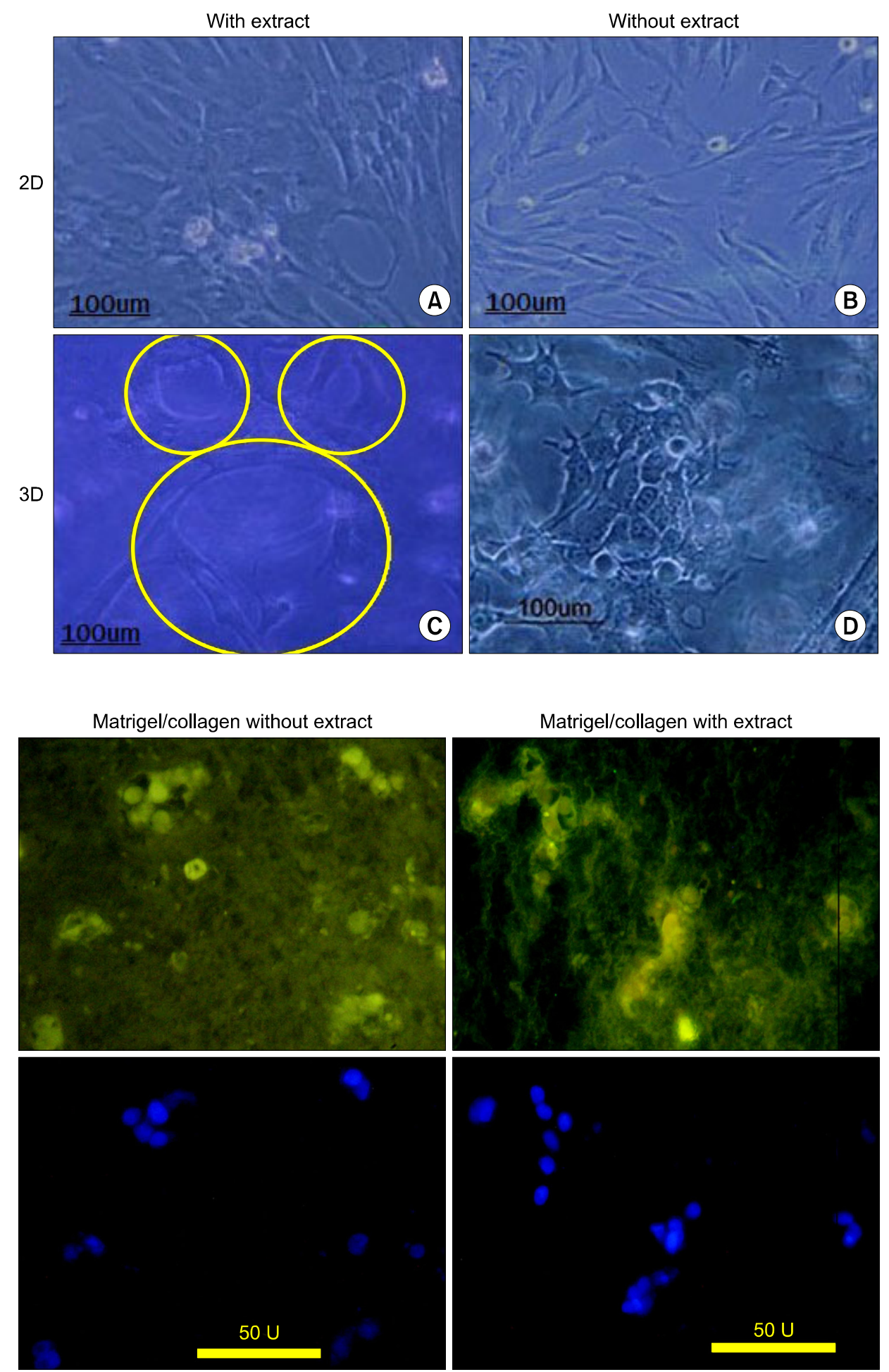

Matrigel/collagen with extract
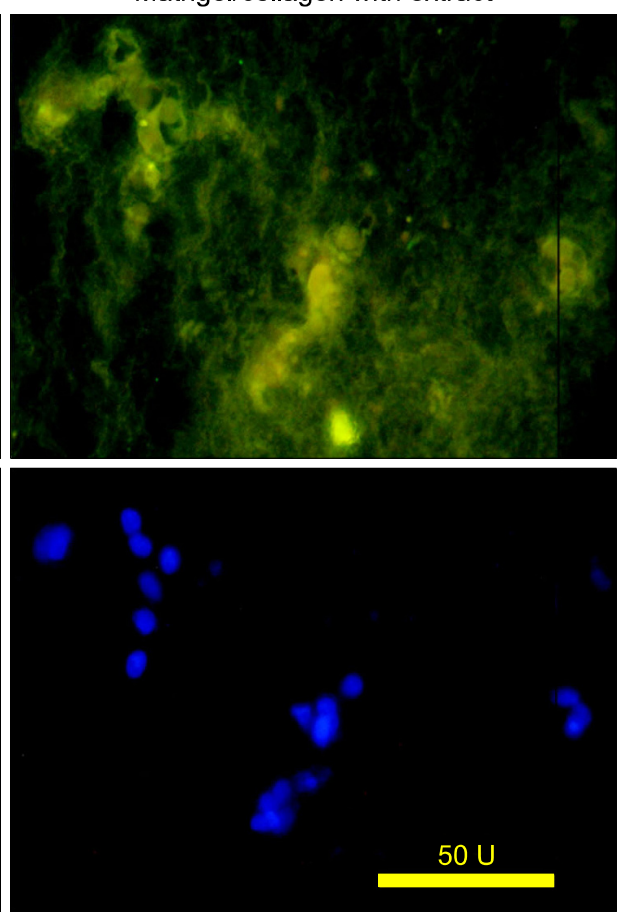

Fig. 2. The Figure shows the morphology of HWJMSCs after supplementation with or without extract for 7 days in 2D culture (A, B). The effects of matrigel/collagen scaffold and fetal liver extract on the cell morphology $(C, D)$. Some cells are arranged into the structure with tubular shape in matrigel/collagen scaffold with or without extract.

Fig. 3. Immunofluorescence micrographs show the HWJMSCs co-cultured with HUVECs expressed albumin in matrigel/collagen scaffold with or without extract, FITC-conjugated albumin (up) and DAPI (down). 


\section{The morphology of HWJMSCs}

Exposing the HWJMSCs with fetal liver extract for 7 days modified the cell phenotype in a polygonal shape (Fig. 2A, B).

The morphology of HWJMSCs was modified from spindle fibroblast- like into a polygonal- and epithelial-like by co-culturing with HUVECs in matrigel/collagen scaffold. The cells also tend to gather and form a luminal shape. The data showed that the presence of the matrigel was critical for phenotypic modifications in HUVECs. Despite the administration of the fetal liver extract, the cell morphology did not modify in the collagen scaffold and the cells kept their spindle or star shapes.

The histology structure of the 3D matrigel/collagen scaffolds containing HWJMSCs and HUVEC showed tubular structures (Fig. 2C, D).

\section{Immunofluorescence}

The data showed both cultures with or without extract expressed albumin (Fig. 3).

Immunohistochemistry of the cells in matrigel/collagen scaffolds with or without extract exhibited a positive reaction for CK19 (Fig. 4). The positive cells were surrounded a luminal space that may indicate they differentiated into cholangiocytes.

\section{Lectin histochemistry}

UEA reacted with endothelial cell in both cultures with or without extract (Fig. 5). Some UEA-positive cells were formed a tubular structure.

Some tubular structures in the cultures exposed to the fetal liver extract were PNA-positive. PNA can detect the bile duct epithelium or cholangiocytes (Fig. 6).
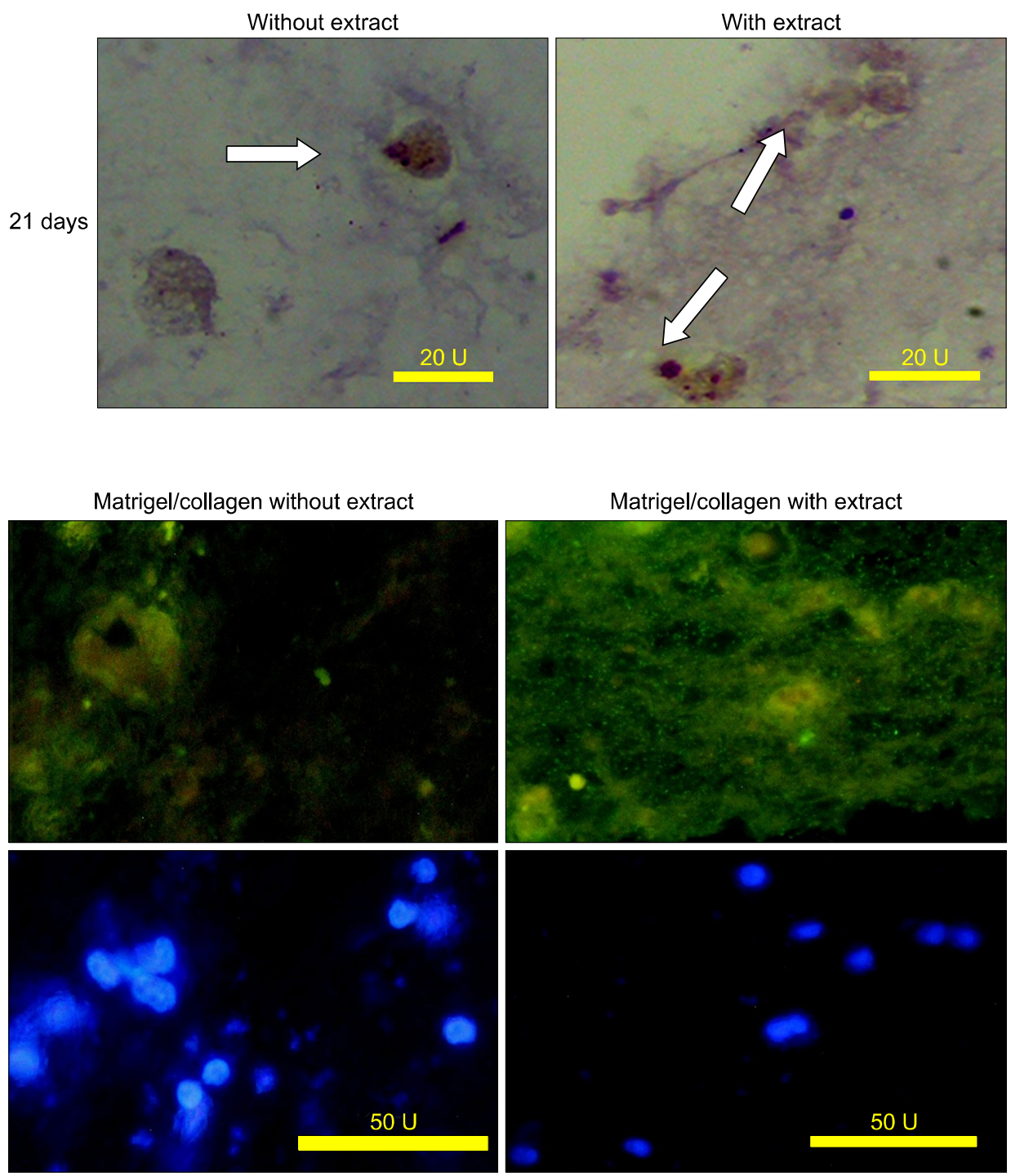

Fig. 4. Immunohistochemical staining of co-culturing HWJMSCs with HUVECs within the matrigel/collagen scaffold with or without extract administration for CK 19.

Fig. 5. The micrographs of the co-cultured cells in the matrigel/collagen scaffold with or without extract stained with lectins UEA. FITC- conjugated lectin (up) and DAPI (down). The unstained cells are non-endothelial cells. 


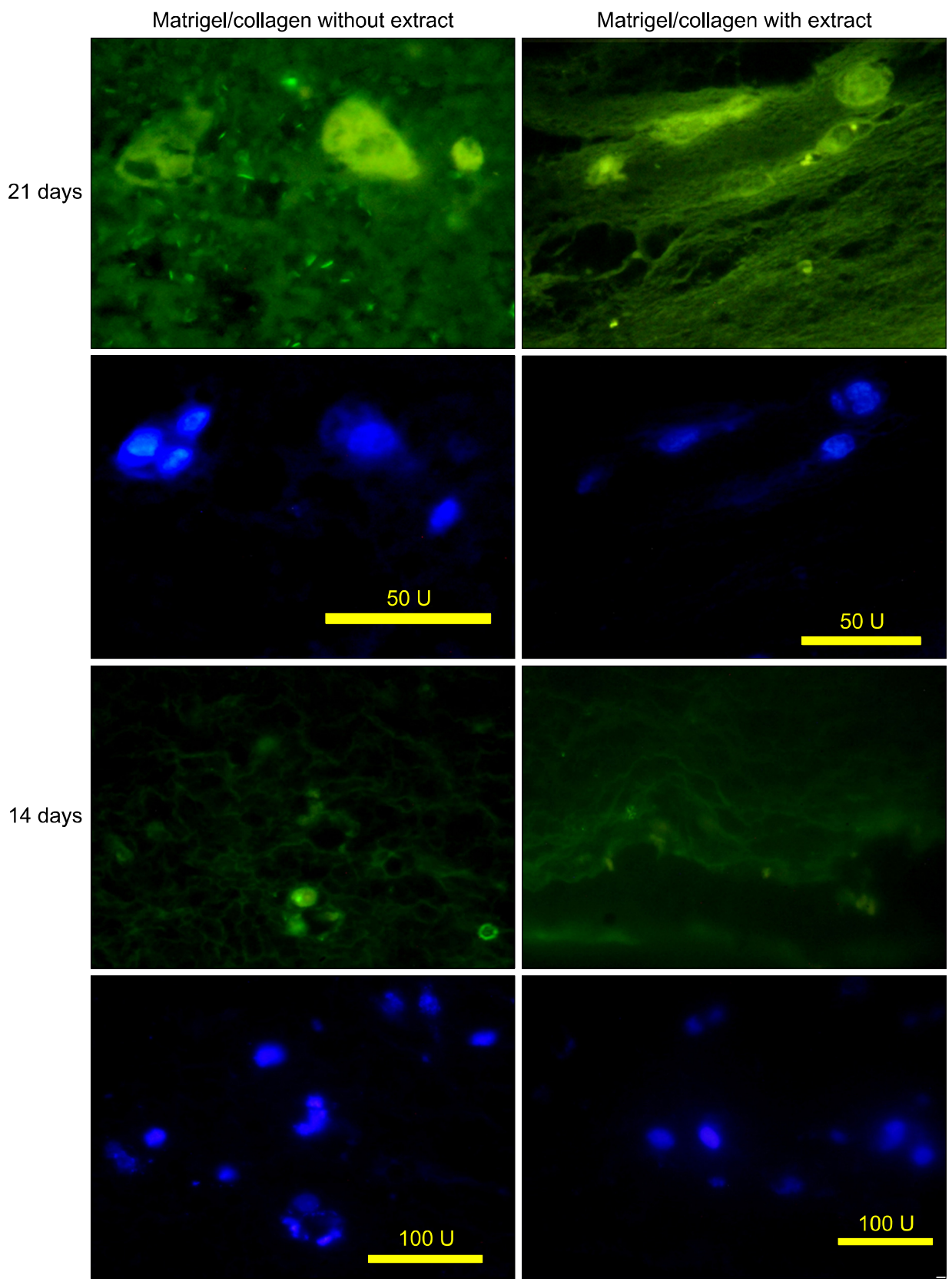

Fig. 6. The micrographs of the bile duct formation in the matrigel/collagen scaffold with or without extract stained with lectins PNA. FITCconjugated lectin (green) and DAPI (blue).

\section{PAS staining}

The cells which surrounded a luminal space were slightly PAS positive and illustrated in Fig. 7.

\section{Discussion}

The cells in 3D culture system showed a different morphological phenotype that could be more similar to the cells located in their normal position. Krause's et al., reported that matrix content seriously affected the cell morphology $(19,20)$. Using matrigel in the collagen scaffolds caused the cells to lose their processes and become round in shape with or without the administration of the extract.

Fetal liver extract includes cell constitutions, liver extracellular matrix, vitamin B12, folic acid, and iron (6). In the last decade, the liver extract has been used as a drug of choice for improving liver functions, treating chronic liver diseases, preventing liver damage, regenerating, proliferating liver tissue, and detoxification. The hematopoietic cells, hepatocytes, hepatic stellate cells and kupffer cells are the sources of ECM in normal and fibrotic liver that can also secret the growth factors such as PDGF and TGF- $\beta$. Fetal liver extract also contains the necessary cytokines for cellular arrangement during liver 

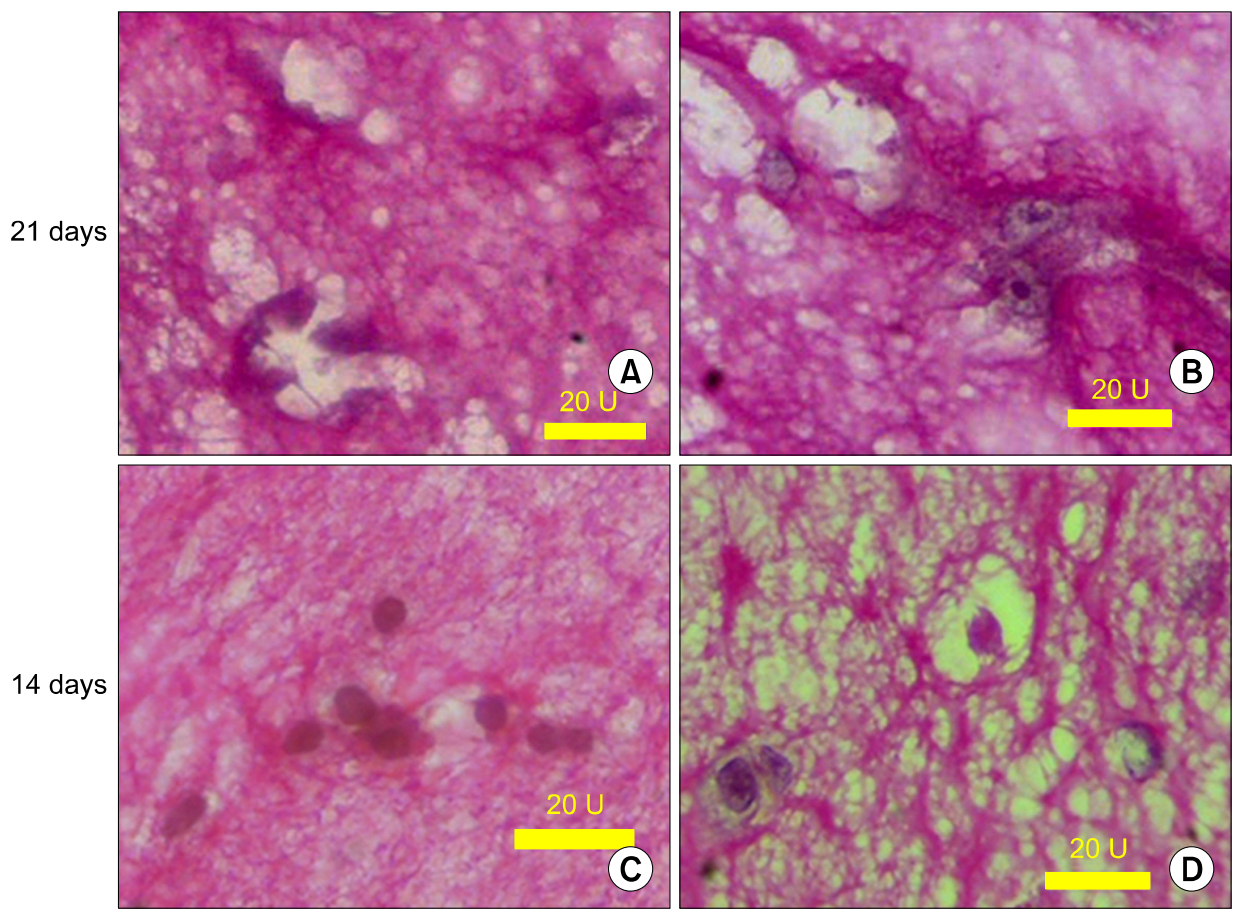

Fig. 7. The light micrograph of 3D culture in matrigel/collagen scaffolds stained with PAS. The luminal space that surrounded by the cells is also depicted; (A\&C) without extract; (B\&D) with extract.

development; therefore, the liver extract components can promote the hepatocytes regeneration and proliferation (21). These components can be responsible for in vitro liver reconstruction.

Hepatocytes differentiation can be improved by co-culturing with the cells found in natural niche. Nagai et al., reported that co-culture of rat non-hepatocytic epithelial with hepatic stellate cell induced hepatocyte differentiation from liver endothelial cell. The stellate cells produce soluble factors that exist in the hepatocyte normal microenvironment; therefore, they play a critical role in stimulating cells toward hepatocytes (22). Co-culturing with hepatic stellate cells also promote hepatic oval cells to differentiate toward hepatocytes. The differentiated cells secreted albumin and expressed HNF-4. They demonstrated a cholangiol-like phenotype (23). It was shown that rat bone marrow MSCs differentiated toward hepatocytes when they were co-cultured with fetal liver cell. Therefore, fetal liver cells formed hepatocyte niche and played an important role in hepatocyte differentiation (24). These results demonstrated that the cells could express CK19, albumin and PNA-containing glycoconjugates in the presence of the extract and endothelial cells within 3D culture condition.

In current study some cells were observed to have a tendency to surround a luminal space. The endothelial cells usually stops their proliferation and becomes tubular in 3D collagen scaffold (25). We used the lectins such as
UEA, as marker of endothelial cells and PNA, as marker of biliary epithelial cells (26). Some PNA and CK19 positive cells were found to surround the luminal spaces. The appearance of CK19, albumin, PNA positive cells within 3D culture was shown in our study. These markers considered as biliary epithelial cells. Data also confirmed another study that showed matrigel/collagen mixture influenced the endothelial cell attachment, migration, differentiation and induction of the tubular shape structure formation (27).

\section{Conclusion}

Co-culturing the stem cells with endothelial cells in 3D Matrigel/collagen mixture showed having the ability to induce the expression of some early liver-specific markers. It may indicate the differentiation of cells toward hepatocyte-like cells, biliary epithelial cells and formation of a tubular shape structures in the presence of fetal liver extract.

\section{Acknowledgments}

This project financially supported by the research deputy of Shiraz University of Medical Sciences and Health services grant No. 6205. Hence, we would like to thank them for their help. The authors would like thank the Research Consultation Center (RCC) of Shiraz University of Medical Sciences for their invaluable assistance in edit- 
ing this article.

\section{Potential conflict of interest}

The authors have no conflicting financial interest.

\section{References}

1. Gaudio E, Carpino G, Cardinale V, Franchitto A, Onori P, Alvaro D. New insights into liver stem cells. Dig Liver Dis 2009;41:455-462

2. Sharma R, Greenhough S, Medine CN, Hay DC. Three-dimensional culture of human embryonic stem cell derived hepatic endoderm and its role in bioartificial liver construction. J Biomed Biotechnol 2010. doi: 10.1155/2010/ 236147.

3. Broutier L, Andersson-Rolf A, Hindley CJ, Boj SF, Clevers $\mathrm{H}$, Koo BK, Huch M. Culture and establishment of self-renewing human and mouse adult liver and pancreas 3D organoids and their genetic manipulation. Nat Protoc 2016;11:1724-1743

4. Handa K, Matsubara K, Fukumitsu K, Guzman-Lepe J, Watson A, Soto-Gutierrez A. Assembly of human organs from stem cells to study liver disease. Am J Pathol 2014; 184:348-357

5. Wang ZZ, Au P, Chen T, Shao Y, Daheron LM, Bai H, Arzigian M, Fukumura D, Jain RK, Scadden DT. Endothelial cells derived from human embryonic stem cells form durable blood vessels in vivo. Nat Biotechnol 2007; 25:317-318

6. Zwicky C, Gerber S, Gasparini D, Forestier F, Hohlfeld P, Tissot JD, Schneider P. Preparation and analysis of fetal liver extracts. Bone Marrow Transplant 2000;26:667-671

7. Kang XQ, Zang WJ, Bao LJ, Li DL, Song TS, Xu XL, Yu XJ. Fibroblast growth factor-4 and hepatocyte growth factor induce differentiation of human umbilical cord blood-derived mesenchymal stem cells into hepatocytes. World J Gastroenterol 2005;11:7461-7465

8. Sellaro TL, Ranade A, Faulk DM, McCabe GP, Dorko K, Badylak SF, Strom SC. Maintenance of human hepatocyte function in vitro by liver-derived extracellular matrix gels. Tissue Eng Part A 2010;16:1075-1082

9. Dawson E, Mapili G, Erickson K, Taqvi S, Roy K. Biomaterials for stem cell differentiation. Adv Drug Deliv Rev 2008;60:215-228

10. Sodek KL, Brown TJ, Ringuette MJ. Collagen I but not Matrigel matrices provide an MMP-dependent barrier to ovarian cancer cell penetration. BMC Cancer 2008;8:223

11. Reed J, Walczak WJ, Petzold ON, Gimzewski JK. In situ mechanical interferometry of matrigel films. Langmuir 2009;25:36-39

12. Machado CB, Ventura JM, Lemos AF, Ferreira JM, Leite MF, Goes AM. 3D chitosan-gelatin-chondroitin porous scaffold improves osteogenic differentiation of mesenchymal stem cells. Biomed Mater 2007;2:124-131

13. Liu H, Roy K. Biomimetic three-dimensional cultures sig- nificantly increase hematopoietic differentiation efficacy of embryonic stem cells. Tissue Eng 2005;11:319-330

14. Dunn JC, Yarmush ML, Koebe HG, Tompkins RG. Hepatocyte function and extracellular matrix geometry: long-term culture in a sandwich configuration. FASEB J 1989;3:174-177

15. Anzalone R, Lo Iacono $M$, Loria $T$, Di Stefano A, Giannuzzi P, Farina F, La Rocca G. Wharton's jelly mesenchymal stem cells as candidates for beta cells regeneration: extending the differentiative and immunomodulatory benefits of adult mesenchymal stem cells for the treatment of type 1 diabetes. Stem Cell Rev 2011;7:342-363

16. Vojdani Z, Khodabandeh Z, Jaberipour M, Hosseini A, Bahmanpour S, Talaei-Khozani T. The influence of fibroblast growth factor 4 on hepatogenic capacity of Wharton's jelly mesenchymal stromal cells. Rom J Morphol Embryol 2015;56:1043-1050

17. LeCouter J, Moritz DR, Li B, Phillips GL, Liang XH, Gerber HP, Hillan KJ, Ferrara N. Angiogenesis-independent endothelial protection of liver: role of VEGFR-1. Science 2003;299:890-893

18. Si-Tayeb K, Lemaigre FP, Duncan SA. Organogenesis and development of the liver. Dev Cell 2010;18:175-189

19. Krause S, Maffini MV, Soto AM, Sonnenschein C. A novel $3 \mathrm{D}$ in vitro culture model to study stromal-epithelial interactions in the mammary gland. Tissue Eng Part C Methods 2008; 14:261-271

20. Li L, Lu Y. Optimizing a 3D culture system to study the interaction between epithelial breast cancer and its surrounding fibroblasts. J Cancer 2011;2:458-466

21. Dan YY, Yeoh GC. Liver stem cells: a scientific and clinical perspective. J Gastroenterol Hepatol 2008;23:687-698

22. Nagai H, Terada K, Watanabe G, Ueno Y, Aiba N, Shibuya T, Kawagoe M, Kameda T, Sato M, Senoo H, Sugiyama T. Differentiation of liver epithelial (stem-like) cells into hepatocytes induced by coculture with hepatic stellate cells. Biochem Biophys Res Commun 2002;293:1420-1425

23. Chen L, Chen XP, Zhang W, Liang HF, Lin YZ, Dong HH, Zhou QD. Differentiation of hepatic oval cell into mature hepatocyte induced by hepatic stellate cells. Zhonghua Gan Zang Bing Za Zhi 2009;17:765-770

24. Lange C, Bruns H, Kluth D, Zander AR, Fiegel HC. Hepatocytic differentiation of mesenchymal stem cells in cocultures with fetal liver cells. World J Gastroenterol 2006;12:2394-2397

25. Kleinman HK, Martin GR. Matrigel: basement membrane matrix with biological activity. in Seminars in cancer biology, Elsevier; 2005.

26. Indramanee S, Silsirivanit A, Pairojkul C, Wongkham C, Wongkham S. Aberrant glycosylation in cholangiocarcinoma demonstrated by lectin-histochemistry. Asian Pac J Cancer Prev 2012;13 Suppl:119-124

27. Donovan D, Brown NJ, Bishop ET, Lewis CE. Comparison of three in vitro human 'angiogenesis' assays with capillaries formed in vivo. Angiogenesis 2001;4:113-121 